\title{
REFINEMENT PROPAGATION Towards Automated Construction of Visual Specifications
}

\author{
Irina Rychkova, Alain Wegmann \\ School of Communication and Computer Science, École Polytechnique Fédérale de Lausanne (EPFL), CH-1015 Lausanne, Switzerland \\ irina.rychkova@epfl.ch, alain.wegmann@epfl.ch
}

Keywords: visual specification, model transformation, stepwise refinement, refinement calculus, refinement propagation.

Abstract: Creation and transformation of visual specifications is driven by modeler's design decisions. After a design decision has been made, the modeler needs to adjust the specification to maintain its correctness. The number of adjustments might make the design process tedious for large specifications. We are interested in techniques that will reduce the modeler's obligation to control specification correctness.

Every single transformation of the visual specification can be captured by the notion of refinement used in formal methods. In this work we present the technique that supports a stepwise refinement of visual specifications based on calculations. We use refinement calculus as a logic for reasoning about refinement correctness. When a design decision is made by the modeler, the necessary adjustments are calculated based on rules of refinement propagation. Refinement propagation can automate the specification adjustment and enforce its correctness.

\section{INTRODUCTION}

It is well accepted by now that visual models play an important role in the information system development. With the growth of system complexity, automated refinement, and refinement verification of visual models is of particular interest.

Stepwise refinement is a well-known paradigm for semantic program constructions originally proposed by Dijkstra (1971) and Wirth (1971). It is based on the idea that a program can be developed through a sequence of refinement steps starting from an abstract specification.

In contrast to techniques where a refinement is first proposed and then proved to be correct, some techniques allow calculation of refinement step based on the refinement laws (Morgan and Gardiner, 1990). The refinement calculus is an underlying theory. This calculation assures refinement correctness 'by construction', and enables the reduction of proof obligations. We believe that refinement by calculation can be beneficial for the practical application in the context of visual modeling.

In this work we introduce a formal semantics for SEAM visual specifications (Wegmann, 2003) using higher-order logic and Refinement Calculus (Back and von Wright, 1998). Based on this semantics, we define a refinement propagation technique that supports a stepwise refinement of visual specifications. We constrained our discussion to the deterministic specifications. The refinement propagation technique is grounded on the following observation: An arbitrary refinement may cause a conflict between model elements. To resolve such a conflict and maintain correctness, model adjustment (also considered as a refinement) is usually required. When the initial refinement can be identified with a design decision that is proposed by a modeler, the adjustment of the entire specification can be calculated based on rules of refinement propagation. Propagation means a sequential application of these rules until saturation. We show that sufficient part of calculations can be done without modeler's involvement. We also specify the situations when modeler's decision is required to accomplish the calculation.

This paper is organized as follows. In Section 2 we present SEAM visual language and classify refinements accepted by this language. In Section 3 we define a formal semantics for SEAM using higherorder logic and refinement calculus. This formaliza- 
tion allows for reasoning about visual specifications with mathematical precision. In Section 4 we introduce the refinement propagation technique. This technique is formulated as eight rules of refinement propagation. In Section 5 we discuss related works. Section 6 presents our conclusions.

\section{REFINEMENT IN 'SEAM' VISUAL MODELING LANGUAGE}

SEAM (Systemic Enterprise Architecture Methodology)(Wegmann, 2003) is an approach for modeling general systems, including information systems and enterprises. SEAM epistemological principles are based on General System Thinking (GST) (Weinberg, 1975) and Living Systems Theory (LST) (Miller, 1995). SEAM ontology is grounded on the second part of RM-ODP (1995) specification. Based on this standard, the main modeling concepts such as object, state, action are defined (Wegmann and Naumenko, 2001). Figure 1 illustrates the SEAM visual notation.

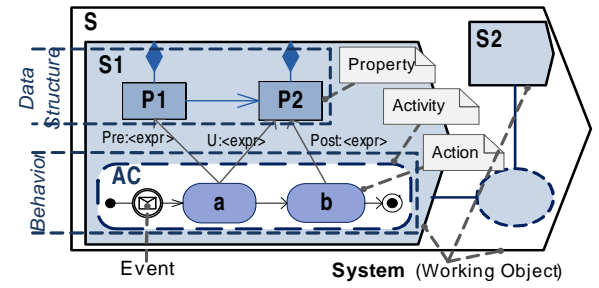

Figure 1: SEAM visual notation.

Any system or system component in SEAM is modeled as a working object. The working object may communicate with an environment by events. Working object $S$ is modeled as a collaboration of two components $S 1$ and $S 2$ (also considered as working objects). $S 1$ is described by its observable properties $P 1, P 2$, and a behavior. Properties constitute the data structure of working object $S 1$ and define its state space. The behavior is represented by a set of actions $a, b$ organized within activity $A C$.

We focus on the refinement of a black box system specification.

Refinement of the state space: ( see also data refinement (Börger, 2003; Woodcock and Davies, 1996; Back, 1989) or data structure refinement (Broy, 1993)) deals with the transformation of system data structure. We recognize the following ways to refine a state space:

- Extension: new property is introduced into the system.

- Reduction: some property is eliminated.
Other refinements are used in visual modeling (e.g. substitution of one property (or group of properties) by another property (or group), property renaming, etc.). It is not difficult to show that these refinements can be represented by a combination of extensions and reductions. Refinement of the system state space is illustrated in Figure 2.

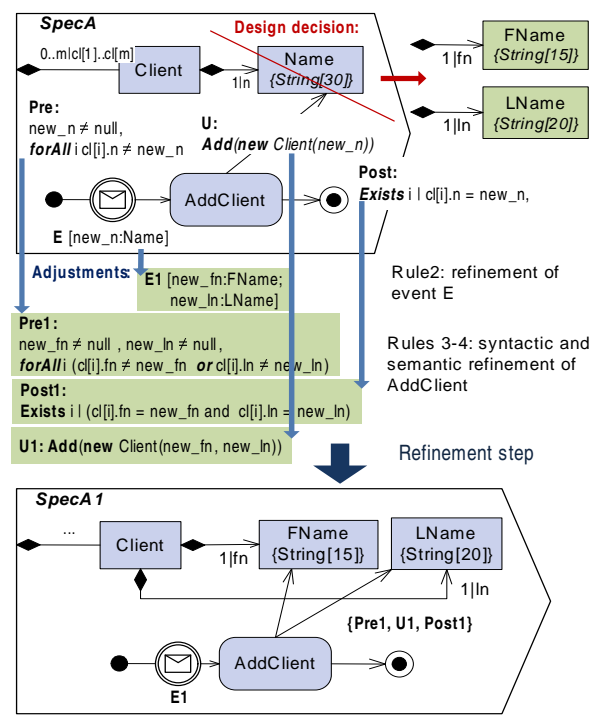

Figure 2: Propagation of refinement of the system state space. Design decision: to substitute Name with $\{$ FName, LName $\}$. Required adjustments made by refinement propagation. A correct SpecA1 is calculated as a result of the refinement step.

\section{Refinement of the behavior:}

- Syntactic Refinement: a number of action's input and output parameters and their types are changed ${ }^{1}$.

- Semantic Refinement: a precondition, an update statement, and/or a postcondition of an action are changed.

- Extension: new behavior (action or activity) is introduced into the system.

- Reduction: some behavior (action or activity) is eliminated.

- Behavior distribution: transition from an action view to an activity (Figure 3).

Aforementioned refinements specify the basis refinement types for SEAM visual specifications. Any arbitrary refinement can be represented as a combination of the basis refinement types. Refinement of the state space and refinement of the behavior can appear

\footnotetext{
${ }^{1}$ This version of behavioral refinement is also called refinement of a syntactic interface (Broy, 1993).
} 


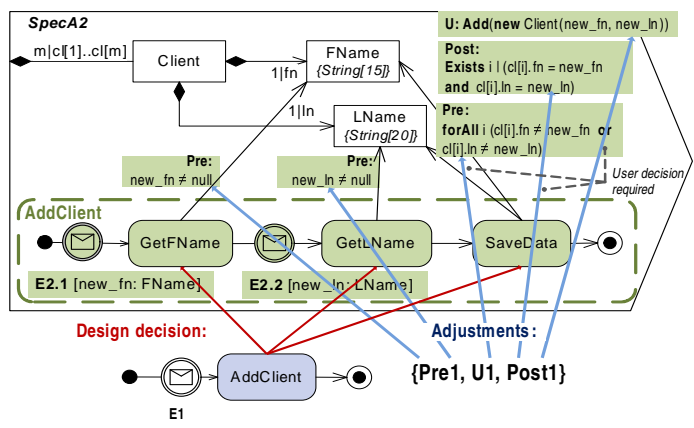

Figure 3: Propagation of behavior distribution refinement. SpecA2 refines SpecA1 (Figure 2) providing a realization of action AddClient.

together in a specification. Also the former can imply the latter or vice versa. This effect we call a refinement propagation.

\section{FORMAL SEMANTICS FOR 'SEAM'}

To reason about refinement with mathematical precision, we formalize SEAM modeling concepts using a higher-order logic and refinement calculus (Back, 1978; Back and von Wright, 1998).

In our work, we were inspired by the ideas presented by Mikhajlova and Sekerinski (1997), Back et al. (2000), and Michajlova (1998). In these works, refinement calculus is used for the formalization of object-oriented program development.

We find it necessary to introduce some concepts of refinement calculus in this section.

\subsection{Introduction of Refinement Calculus}

In this paper we restrict our study to deterministic specifications. Nondeterminism will be addressed in our future work.

A program state in refinement calculus is modeled as a tuple of values of all program components. A program state space (a type) $\Sigma$ is defined as a cartesian product $\Sigma=\Sigma_{1} \times \Sigma_{2} \times \ldots \times \Sigma_{n}$ where $\Sigma_{1}, \Sigma_{2}, \ldots, \Sigma_{n}$ are state spaces of all program components.

A predicate over a state space $\Sigma$ is a boolean function. The set of all predicates defined over the state space $\Sigma$ is denoted by

$$
\mathcal{P \Sigma} \hat{=} \Sigma \rightarrow\{\text { true }, \text { false }\}
$$

A relation between two state spaces $\Sigma$ and $\Gamma$ is a function that maps each state in $\Sigma$ into a predicate in $\Gamma$. The set of all relations from $\Sigma$ to $\Gamma$ is denoted by

$$
\Sigma \leftrightarrow \Gamma \hat{=} \Sigma \rightarrow P \Gamma
$$

It is equivalent to another, more familiar definition: $\Sigma \leftrightarrow \Gamma \hat{=}(\Sigma \times \Gamma) \rightarrow\{$ true, false $\}$

A predicate transformer from $\Sigma$ to $\Gamma$ is a function that maps each predicate in $\Sigma$ into a predicate in $\Gamma$.
The set of all predicate transformers from $\Sigma$ to $\Gamma$ is denoted by

$$
\Sigma \mapsto \Gamma \hat{=} \mathcal{P} \Sigma \rightarrow \mathcal{P} \Gamma
$$

Predicate transformer $\langle f\rangle$ is called a functional update. It applies the function $f$ to a state $\sigma$ to yield a new state $f$. $\sigma$. For all states $\sigma$, defined by the precondition $p$, the functional update will produce the state $f \sigma$, for which the postcondition $q$ holds:

$$
\forall \sigma \in \Sigma \mid p \bullet\langle f\rangle . q . \sigma \hat{=} q(f \sigma)
$$

Program statements in refinement calculus are identified with predicate transformers.

For a sequential composition of statements $S, T$, and predicates $p, q, r$ :

$$
p\{|S ; T|\} q \equiv(\exists r \bullet p\{|S|\} r \wedge r\{|T|\} q
$$

A refinement ordering on the predicate transformers is defined as follows. For $S, T: \Sigma \mapsto \Gamma$ :

$$
S \sqsubseteq T \hat{=}(\forall q: P \Gamma \bullet S . q \subseteq T . q)
$$

For refinement ordering the following holds:

$$
\begin{gathered}
S_{1} \sqsubseteq S_{1}^{\prime} \wedge S_{2} \sqsubseteq S_{2}^{\prime} \Rightarrow\left(S_{1} \times S_{2}\right) \sqsubseteq\left(S_{1}^{\prime} \times S_{2}^{\prime}\right) \\
S \sqsubseteq S^{\prime} \sqsubseteq S^{\prime \prime} \Rightarrow S \sqsubseteq S^{\prime \prime}
\end{gathered}
$$

\subsection{Formalization of SEAM Modeling Concepts}

We distinguish between the following views of working objects in SEAM:

- Working object as a whole $W O_{\text {whole }}$ - a black box system specification;

- Working object as a composite $W O_{\text {composite }}$ - a white box system specification.

In this paper, we focus on the black box specifications.

Working object as a whole describes the system by a number of properties $P_{1} \ldots P_{m}$ that specify data types, and a behavior $\mathcal{B}$.

We declare the working object $W O_{\text {whole }}$ as follows:

$$
\begin{aligned}
& W O_{\text {whole }} \hat{=} \\
& \quad p_{1_{1}}, . . p_{n_{1}}: P_{1}, \\
& \quad \ldots, \\
& p_{1_{m}}, . . p_{n_{m}}: P_{m},
\end{aligned}
$$

where $p_{1_{i}}, . . p_{n_{i}}$ are instances of a property $P_{i}$.

Working objects may interact with the environment by receiving inputs $I_{1}, . ., I_{k}$ and sending outputs $O_{1}, . . O_{l}$, also modeled as a part of the system.

We distinguish between primitive and compound properties. The former can be considered as an alias for an operational data type (e.g. Int, String, Boolean, etc.); the latter is defined by a set of component properties and references to properties using propertyproperty relations. 
Example 1. SpecA in Figure 2 specifies a working object SpecA $A_{\text {whole }}$ with its properties Client and Name. Property Client is compound, it has a component property Name. Name is primitive - an alias for strings of length 30 . This representation unifies a declarative specification style:

"..client is identified by his/her name (30 symbols).." and an imperative style, intrinsic to programming: class Client \{ name: String[30]; \} // String of length 30 $\mathrm{m}$ : Int; // number of clients $\mathrm{cl}$ : Array(m) of Client;// list of clients

A state of the primitive property denotes a value of the corresponding operational type (e.g. 1 ,"ABC",true); a state of the compound property is defined by the states of its components and references. A tuple of property instances $p_{1_{1}} . . p_{n_{m}}$, inputs $i_{1_{1}} . . i_{n_{k}}$, outputs $o_{1_{1}} . . o_{n_{l}}$ and their corresponding states defines a system state $\sigma \in \Sigma$.

$\Sigma$ specifies a system state space - a set of all possible states of the working object:

$$
\begin{aligned}
\Sigma & \hat{=} \Sigma_{P} \times \Sigma_{I n} \times \Sigma_{\text {Out }} \quad \text { where } \\
\Sigma_{P} & =(\underbrace{\Sigma_{P_{1}} \times \ldots \times \Sigma_{P_{1}}}_{n_{1}}) \times \ldots \times(\underbrace{\Sigma_{P_{m}} \times \ldots \times \Sigma_{P_{m}}}_{n_{m}}) \\
\Sigma_{I n} & =(\underbrace{\Sigma_{I_{1}} \times \ldots \times \Sigma_{I_{1}}}_{n i_{1}}) \times \ldots \times(\underbrace{\Sigma_{I_{k}} \times \ldots \times \Sigma_{I_{k}}}_{n i_{k}}) \\
\Sigma_{\text {Out }} & =(\underbrace{\Sigma_{O_{1}} \times \ldots \times \Sigma_{O_{1}}}_{n 0_{1}}) \times \ldots \times(\underbrace{\Sigma_{O_{l}} \times \ldots \times \Sigma_{O_{l}}}_{n o_{l}})
\end{aligned}
$$

$\Sigma_{P}, \Sigma_{I n}, \Sigma_{\text {Out }}$ denote state spaces of system properties, inputs, and outputs respectively.

Example2. State space of the working object SpecA1 in Figure 2:

$$
\begin{aligned}
& \Sigma_{\text {SpecA1 }}=\underbrace{\Sigma_{\text {Client }} \times \ldots \times \Sigma_{\text {Client }}}_{m} \times \Sigma_{\text {In }} \Rightarrow \\
& \Sigma_{\text {SpecA1 }}=\underbrace{(\text { String }[15] \times \text { String }[20]) \times \ldots \times(. .)}_{m+1}
\end{aligned}
$$

Behavior $\mathcal{B}$ of a working object can be seen as an action or as an activity.

Action $A$ is defined by a three-tuple $\{$ Pre, $U$, Post $\}$. Pre-condition Pre and post-condition Post define the states of the system $\sigma, \sigma^{\prime} \in \Sigma$ before and after the action respectively. An update $U$ specifies a transition from pre- state to post- state.

If this action describes a communication with the environment, the input and output events $E_{\text {In }}, E_{\text {Out }}$ also make part of the action specification ${ }^{2}$.

\footnotetext{
${ }^{2}$ Broy (1993) defines input and output channels and the sort of messages for each channel as a syntactic interface of the black box system view. The causal relationship between input messages and output messages is defined as a semantic interface of this view.
}

$$
\begin{aligned}
A \hat{=} & \\
& E_{\text {In }}\left(I_{1}, . ., I_{k}\right), \quad E_{\text {Out }}\left(O_{1}, . ., O_{l}\right), \\
& \text { Pre }: \mathcal{P}\left(\Sigma_{P} \times \Sigma_{\text {In }}\right), \\
& U:\left(\Sigma_{P} \times \Sigma_{\text {In }}\right) \rightarrow\left(\Sigma_{P} \times \Sigma_{\text {Out }}\right) \\
& \text { Post }: \mathcal{P}\left(\Sigma_{P} \times \Sigma_{\text {Out }}\right),
\end{aligned}
$$

For the deterministic specifications, we consider a functional update $\langle U\rangle: \Sigma \mapsto \Sigma$. $U$ is a function that calculates a post-state from the pre-state. Using the definition of functional update from (4), we can write:

$$
\forall \sigma \in \Sigma \mid \text { Pre } \bullet\langle U\rangle \text {.Post. } \sigma \hat{=} \text { Post }(U \sigma)
$$

We specify action $A$ with its precondition Pre, functional update $\langle U\rangle$ and postcondition Post as follows:

$$
\begin{gathered}
\text { Pre }\{|A|\} \text { Post } \hat{=} \\
\text { Pre }\{|\langle U\rangle|\} \text { Post } \equiv \text { Pre } \subseteq\langle U\rangle^{-1} \text {.Post }
\end{gathered}
$$

Example3. SpecA in Figure 2 defines an action AddClient:

AddClient $=$

$E_{\text {In }}($ Name $)$,

Pre $=\exists$ new $n:$ Name $\wedge \forall$ cl $\bullet$ cl. $n \neq$ new $\_n$

$U: \Sigma_{\text {Client }} \rightarrow \Sigma_{\text {Client }}=\operatorname{Add}($ newClient $($ new_n $))$

Post $=\exists c l \mid c l . n=$ new $_{n}$

Pre states that there exists an input parameter $n e w \_n$ and that there is no client with the attribute $n=n e w \_n$ presented in the system. $U$ denotes that a new instance of client newClient $\left(n e w \_n\right)$ is added into the system. The postcondition Post specifies the fact that after AddClient is carried out, the client with the attribute $n=n e w \_n$ does exist in the system. $E_{I n}$ (Name) specifies the input event $E$, that transmits a parameter new $n$. $\square$

Activity $A c$ can be considered as a detailed specification of action $A$ : it describes how the transition from pre- state to post- state is performed. Ac defines a set of component actions and the way they are composed to carry out the transition:

$$
A c \hat{=} A_{1} \bigcirc A_{2} \bigcirc \ldots \bigcirc A_{t}
$$

where $\bigcirc$ stands for component action ordering, defined by a corresponding action-action relation.

Precondition Pre, update $U$, and postcondition Post of $A$ are related to those of component actions:

$$
\begin{aligned}
& \text { Pre }_{A}=\rho_{\text {pre }}\left(\text { Pre }_{A_{1}}, . \text { Pre }_{A_{t}}\right) ; \\
& U_{A}=\rho_{u}\left(U_{1}, \ldots U_{t}\right) ; \\
& \text { Post }_{A}=\rho_{\text {post }}\left(\text { Post }_{A_{1}}, \ldots, \text { Post }_{A_{t}}\right) ;
\end{aligned}
$$

$\rho_{\text {pre }}, \rho_{u}, \rho_{\text {post }}$ are defined by the component action ordering $(\bigcirc)$.

Input and output events $E_{I n}, E_{\text {Out }}$ of the activity $A c$, are related to the input and output events of component actions $A_{1}, . . A_{t}$ :

$$
\Sigma_{\text {In } / \text { Out }} \subseteq \Sigma_{\text {In } / \text { Out }_{1}} \times \ldots \times \Sigma_{\text {In }_{\text {Out }}}
$$

Example4. SpecA2 in Figure 3 defines an activity AddClient that specifies the realization of the action 
AddClient from SpecA1, Figure 2. Parameters of an input event $E$ are distributed between input evens $E_{2.1}$ and $E_{2.2}$ of component actions GetFName and GetLName such that:

$E($ FName, LName $) \equiv\left\{E_{2.1}(\right.$ FName $), E_{2.2}($ LName $\left.)\right\}$ and $\Sigma_{\text {In }} \equiv\left(\Sigma_{\text {FName }} \times \Sigma_{\text {LName }}\right)$

\section{REFINEMENT PROPAGATION}

In this section we introduce the refinement propagation technique for visual specifications. Exploring the basis refinement types, specified in section 2, we found relations between them in the form "refinementX implies refinement $Y$ ". This implication we call a propagation of refinement.

\subsection{Definition of Refinement for Visual Specifications}

Refinement calculus specifies the correct refinement between program statements as a refinement ordering on the predicate transformers. The program statement $S$ is said to be correctly refined by the program statement $T$, iff $T$ satisfies any specification statement satisfied by $S$, (6).

The specification statement expresses requirements program has to meet in a given state. Definition of refinement for the programm statements (or programs) can be generalized for visual specification statements (or visual specifications) as follows:

Definition 1. Specification WO (abstract) is correctly refined by specification $W O^{\prime}$ (concrete) $W O \sqsubseteq W O^{\prime}$, if $W O^{\prime}$ satisfies any requirement satisfied by $\bar{W} O$.

\subsection{Propagation Rules}

In refinement calculus, refinement ordering is monotonic with respect to the sequential statement construction. It means that if $S$ is a statement and $S_{1}$ occurs as a substatement, $S=S\left(S_{1}\right)$ then

$$
S_{1} \sqsubseteq S_{1}^{\prime} \Rightarrow S\left(S_{1}\right) \sqsubseteq S\left(S_{1}^{\prime}\right)
$$

We can write $W O_{\text {whole }}$ as a compound statement:

$$
W O_{\text {whole }}\left(P_{1}, . ., P_{m}, I_{1}, . ., I_{k}, O_{1}, . ., O_{l}, \mathcal{B}\right)
$$

For the substatements, the following can be written:

$$
P_{i}=P_{i}\left(. ., P_{j}, . .\right), I_{i}=I_{i}\left(. ., I_{j}, . .\right), O_{i}=\left(. ., O_{j}, . .\right)
$$

where $P_{i}, I_{i}, O_{i}$ may be compound properties; and:

$$
\begin{aligned}
& \mathcal{B}=A\left(E_{\text {In }}, E_{\text {Out }}, \text { Pre }, U, \text { Post }\right) \quad \mid \quad A c\left(A_{1}, . ., A_{t}\right), \\
& E_{\text {In }}=E_{\text {In }}\left(. ., I_{i}, . .\right), E_{\text {Out }}=E_{\text {Out }}\left(. ., O_{i}, . .\right), \\
& \text { Pre }=\operatorname{Pre}\left(. ., I_{i}, . ., P_{j}, . .\right), \text { Post }=\text { Post }\left(. ., P_{i}, . ., O_{j}, . .\right) \\
& U=U\left(. ., I_{i}, . ., P_{j}, . ., O_{k}, . .\right)
\end{aligned}
$$

Properties, events, actions, and activities are related within specification. Thereby a basis refinement of an arbitrary substatement $X$ from (17), (18) may cause a conflict between certain elements, such that

$$
X \sqsubseteq X^{\prime} \wedge W O_{\text {whole }}(X) \nsubseteq W O_{\text {whole }}\left(X^{\prime}\right)
$$

To resolve this conflict, and to preserve monotonicity, refinement $X_{1}^{\prime}$ of some other substatement $X_{1}$ is usually required. This assertion formalizes our definition of refinement propagation. Within a final number of steps, refinement propagation either results in correct refinement of specification $W O_{\text {whole }}$ :

$$
\begin{gathered}
\left(X \sqsubseteq X^{\prime}\right) \Rightarrow\left(X_{1} \sqsubseteq X_{1}^{\prime}\right) \ldots \Rightarrow\left(X_{n} \sqsubseteq X_{n}^{\prime}\right) \Rightarrow \\
W O_{\text {whole }}\left(X, X_{1}, . ., X_{n}\right) \sqsubseteq W O_{\text {whole }}\left(X^{\prime}, X_{1}^{\prime}, . ., X_{n}^{\prime}\right)
\end{gathered}
$$

or indicates such a refinement impossible.

\section{Resolving Conflicts between Properties}

We distinguish between compound and primitive properties. The elimination of a compound property may cause 'free-floating' properties and make the specification confusing.

RULE 1: If $P_{i}\left(P_{i_{1}}, \ldots, P_{i_{k}}, P_{i_{k+1}}, \ldots, P_{i_{l}}\right)$ is a compound property with components $P_{i_{1}} . . P_{i_{k}}$ and references on properties $P_{i_{k+1}} . . P_{i_{l}}$, and, by reduction of the state space, $P_{i}$ is eliminated, then its component properties $P_{i_{1}}, \ldots, P_{i_{k}}$ have to be also eliminated. Reduction of the state space must be correct.

$$
\begin{array}{r}
W O_{\text {whole }}\left(. ., P_{i-1}, P_{i}, P_{i_{1}}, . ., P_{i_{l}}, P_{i+1}, . .\right) \sqsubseteq-P_{i} \\
W O_{\text {whole }}\left(. ., P_{i-1}, P_{i_{k+1}}, . ., P_{i_{l}} P_{i+1}, . .\right)
\end{array}
$$

We formulate the correctness of refinement of the state space:

Definition 2. Let $W O_{\text {whole }}$ be a system specification as defined in (9); $W O_{\text {whole }}^{\prime}$ is its refinement, where some properties have been introduced, eliminated, or substituted; and $r$ is an abstraction relation connecting 'old' and 'new' states:

$$
r: \Sigma_{w o^{\prime}} \leftrightarrow \Sigma_{w o}
$$

The refinement of the state space is correct with respect to $r$, if any state of the abstract specification has a corresponding state (or group of states) of the concrete specification (i.e. $r$ is a total surjective function).

The notion of $r$ can be generalized. Considering $R$ as a relation between predicates of the abstract and concrete specifications, we can write:

$$
\begin{gathered}
R: \Sigma_{w o^{\prime}} \mapsto \Sigma_{w o} \\
W O \sqsubseteq_{R} W O^{\prime} \hat{=} W O \sqsubseteq W O^{\prime} ; R
\end{gathered}
$$

Example5. SpecA in Figure 2 is refined by SpecAl where the property Name is substituted with two other properties FName and LName. To maintain the refinement correctness, we have to define an abstraction relation between 'old' and 'new' data types as a total surjective function:

$$
r: \Sigma_{\text {Client }^{\prime}} \rightarrow \Sigma_{\text {Client }} \Leftrightarrow\left(\Sigma_{\text {FName }} \times \Sigma_{\text {LName }}\right) \rightarrow \Sigma_{\text {Name }}
$$

for example:

$$
\begin{aligned}
& \text { 1. } \mathrm{n}:=\operatorname{substr}(\mathrm{fn}+\mathrm{ln}, 30) \\
& \text { 2. } \mathrm{n}:=\ln / / \mathrm{n} \text { in 'old' }=\ln \text { in 'new' spec } \\
& \text { 3. ... }
\end{aligned}
$$

considering the second expression for $n$, we define: 


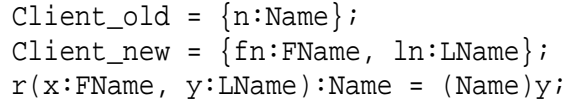

This relation can be generalized as a predicate transformer:

$$
\begin{gathered}
R:\left(\Sigma_{\text {Client' }} \rightarrow\{\text { true }, \text { false }\}\right) \rightarrow\left(\Sigma_{\text {Client }} \rightarrow\{\text { true }, \text { false }\}\right) \\
\Leftrightarrow\left(\Sigma_{\text {FName }} \times \Sigma_{\text {LName }}\right) \mapsto \Sigma_{\text {Name }}
\end{gathered}
$$

This refinement step causes a conflict between refined property Client' and the input event $E$ where the 'eliminated' property Name occurs as a parameter. Thus the event specification has to be adjusted (refined):

$$
\text { Client } \sqsubseteq \text { Client }^{\prime} \Rightarrow E \sqsubseteq E^{\prime}
$$

We generalize this observation as a propagation rule.

\section{Resolving Conflicts between Properties and Events}

RULE 2: If $P^{\prime}$ is a refinement of a property $P$, and $E(. ., P, ..) \hat{=} E(P)$ is an event, where $P$ occurs as a parameter type, i.e. $\Sigma_{P} \subseteq \Sigma_{E}$, then the following holds:

$$
P \sqsubseteq P^{\prime} \Rightarrow E_{\text {In/Out }}(P) \sqsubseteq E_{\text {In } / \text { Out }}\left(P^{\prime}\right)
$$

Example5 (continue): The following elaboration of specification SpecAl is deducible:

$$
\begin{gathered}
\text { Client } \sqsubseteq \text { Client }^{\prime}, \text { where } \\
\Sigma_{\text {Client }}=\Sigma_{\text {Name }}, \Sigma_{\text {Client }^{\prime}}=\Sigma_{\text {FName }} \times \Sigma_{\text {LName }}, \Sigma_{\text {Name }} \subseteq \Sigma_{\text {In }} \\
\Downarrow \\
E(\text { Name }) \sqsubseteq E_{1}(\text { FName }, \text { LName })
\end{gathered}
$$

\section{Resolving Conflicts between Events and Actions: Syntactic Refinement}

RULE 3: If $E$ is an input (output) event of an action $A$, then a refinement of $E$ is a syntactic refinement of the action $A$ (by definition of syntactic refinement). Syntactic refinement must be correct.

$$
E \sqsubseteq E^{\prime} \Leftrightarrow A(E) \sqsubseteq \operatorname{syn}_{E} A\left(E^{\prime}\right)
$$

Definition 3. Let $A^{\prime}$ be a syntactic refinement of an action $A$, and $r_{\text {In }}$ and $r_{O u t}$ are abstraction relations for connecting 'old' input and output parameters with the 'new' ones:

$$
r_{\text {In } / \text { Out }}: \Sigma_{w o^{\prime}} \rightarrow \Sigma_{\text {In } / \text { Out }}
$$

where $\Sigma_{w o^{\prime}}$ is a state space of the refined working object. $R_{\text {In }}, R_{\text {Out }}$ are the corresponding predicate transformers:

$$
\begin{aligned}
& R_{\text {In }}:\left(\Sigma_{w o^{\prime}} \rightarrow \Sigma_{\text {In }}\right) \rightarrow\{\text { true }, \text { false }\}=\Sigma_{w o^{\prime}} \mapsto \Sigma_{\text {In }} \\
& R_{\text {Out }}:\left(\Sigma_{w o^{\prime}} \rightarrow \Sigma_{\text {Out }}\right) \rightarrow\{\text { true }, \text { false }\}=\Sigma_{w o^{\prime}} \mapsto \Sigma_{\text {Out }}
\end{aligned}
$$

$A$ is correctly refined by $A^{\prime}$ with respect to $R_{I n}, R_{O u t}$ if

$$
A \sqsubseteq R_{\text {In }}, R_{\text {Out }} A^{\prime} \hat{=} \quad R_{\text {In }} ; A \sqsubseteq A^{\prime} ; R_{\text {Out }}
$$

$R_{I n}$ and $R_{O u t}$ are total and surjective.

For the propagation Rule 3 we write:

$$
\begin{array}{cll}
E_{\text {In }} \sqsubseteq E_{\text {In }}^{\prime} & \Leftrightarrow & R_{\text {In }} ; A(E) \sqsubseteq A\left(E^{\prime}\right) \\
E_{\text {Out }} \sqsubseteq E_{\text {Out }}^{\prime} & \Leftrightarrow & A(E) \sqsubseteq A\left(E^{\prime}\right) ; R_{\text {Out }}
\end{array}
$$

Example6. For the action AddClient in Figure 2, syntactic refinement is correct if predicate transformer $R_{I n}$ is defined:

$$
R_{\text {In }}: \Sigma_{\text {SpecA } 1} \mapsto \Sigma_{\text {Client }}=\left(\Sigma_{\text {FName }} \times \Sigma_{\text {LName }}\right) \mapsto \Sigma_{\text {Name }}
$$

The definition of $R_{I n}$ is a modeler's choice. Here we take $R_{I n} \equiv R$, as it was specified in Example5.

$E($ Name $) \sqsubseteq E 1($ FName, LName $) \Rightarrow$

$R_{\text {In }} ;$ AddClient $(E$ (Name $\left.)\right) \sqsubseteq$ AddClient $(E 1$ (FName, LName $\left.)\right)$

\section{Resolving Conflicts between Events and Actions: Semantic Refinement}

RULE 4: If $E$ is an input (output) event of an action $A$, and $E$ or some of its parameters $I_{i}\left(O_{i}\right)$ occurs in Pre, $U$, or Post of $A$, then a refinement of $E$ implies a semantic refinement of the action $A$. Semantic refinement must be correct.

$$
\begin{gathered}
A \mid \operatorname{Pre}(E) \vee U(E) \vee \operatorname{Post}(E) \Rightarrow \\
E \sqsubseteq E^{\prime} \Rightarrow A \sqsubseteq \operatorname{sem}_{E} A^{\prime}
\end{gathered}
$$

Definition 4. Let $A$ be an action, defined by the tuple $\{E$, Pre,$U$, Post $\} ; A^{\prime}:\left\{E^{\prime}\right.$, Pre $^{\prime}, U^{\prime}$, Post $\left.^{\prime}\right\}$ is its semantic refinement, where $P r e^{\prime}$, Post $^{\prime}$ are new preand postconditions, and $U^{\prime}$ is a new update. $A$ is correctly refined by $A^{\prime}$ if:

- $A^{\prime}$ is applicable at least on every state where $A$ is applicable;

- starting at the corresponding initial states, $A^{\prime}$ and $A$ produce equivalent results.

Providing abstraction relation $R$, which relates 'old' and 'new' predicates (Definition 2), and using action specification from (12), $A$ is said to be correctly refined by $A^{\prime}$ with respect to $R$ if

$$
\text { R.Pre } \subseteq\langle U\rangle^{-1} \text {.Post and Pre } \subseteq\left(\left\langle U^{\prime}\right\rangle^{-1} ; R\right) \text {. Post }{ }^{\prime}
$$

For the propagation Rule 4 we write:

$$
E \sqsubseteq E^{\prime} \Rightarrow R ; A \sqsubseteq\left(E^{\prime} / E\right) A ; R
$$

where $\left(E^{\prime} / E\right) A$ stands for a substitution of each occurrence of $E$ or one of its parameters by $E^{\prime}$, or its corresponding parameter.

Example7. SpecAl illustrates the semantic refinement of the action AddClient, specified in Example3:

$$
\begin{aligned}
& \text { AddClient }{ }^{\prime}= \\
& E_{1} \text { (FName, LName), } \\
& \mathrm{Pre}_{1}=\exists \text { new_fn: FName, new_ln: LName } \wedge \\
& \forall c l \bullet\left(c l . f n \neq n e w_{-} f n \vee c l . l n \neq n e w_{-} l n\right) \\
& U_{1}: \Sigma_{\text {Client }} \rightarrow \Sigma_{\text {Client }}=\operatorname{Add}\left(\text { newClient }\left(\text { new }_{-} \text {fn,new_ln }\right)\right) \\
& \text { Post }_{1}=\exists c l \mid\left(c l . f n=n e w_{-} f n \wedge c l . l n=n e w_{-} l n\right)
\end{aligned}
$$


New precondition Pre $_{1}$ and postcondition Post $_{1}$ have been calculated by propagation:

$$
\begin{aligned}
& \left.E(\text { Name }) \sqsubseteq E_{1}(\text { FName }, \text { LName }) \Rightarrow \text { (rule } 4\right) \\
& \operatorname{AddClient}(E) \sqsubseteq_{\operatorname{sem}_{E}} \text { AddClient }\left(E_{1}\right) \\
& \text { Pre }=\text { Pre }(\text { Name }), \text { Post }=\text { Post }(\text { Name }) \Rightarrow(\text { rule 5) } \\
& \text { AddClient(Pre,Post) } \text { sem }_{E} \text { AddClient }\left(\text { Pre }_{1}, \text { Post }_{1}\right) \text {, and } \\
& \text { Pre }_{1}=\exists \text { new } f n: \text { FName, new_ln }: \text { LName } \wedge \\
& \forall c l \bullet\left(c l . f n \neq n e w_{-} f n \vee c l . l n \neq n e w_{-} l n\right) \\
& \text { Post }_{1}=\exists c l \mid\left(\text { cl.fn }=\text { new }_{-} f n \wedge \text { cl.ln }=\text { new_ln }\right)
\end{aligned}
$$

This refinement is correct by Definition 4. For the sake of brevity, the proof of correctness is omitted. The specification adjustment is finished and the refinement propagation is complete.

$$
\begin{gathered}
\left(\text { Client } \sqsubseteq \text { Client }^{\prime}\right) \Rightarrow\left(E \sqsubseteq E_{1}\right) \Rightarrow(\text { AddCl.. } \sqsubseteq \text { AddCl.. }) \Rightarrow \\
\text { SpecA }(\text { Name }, E, \text { AddClient }) \sqsubseteq \\
\text { SpecA } 1\left(\{\text { FName }, \text { LName }\}, E_{1}, \text { AddClient }^{\prime}\right) \quad \square
\end{gathered}
$$

\section{Resolving Conflicts between Properties and Actions: Semantic Refinement}

RULE 5: If property $P$ occurs in Pre, $U$, or Post of action $A$, then a refinement of $P$ implies a semantic refinement of $A$. Semantic refinement must be correct.

$$
\begin{gathered}
A \mid \operatorname{Pre}(P) \vee U(P) \vee \operatorname{Post}(P) \Rightarrow \\
P \sqsubseteq P^{\prime} \Rightarrow A \sqsubseteq_{\operatorname{sem}_{P}} A^{\prime}
\end{gathered}
$$

By definition of correct semantic refinement (Definition 4), we write:

$$
P \sqsubseteq P^{\prime} \Rightarrow R ; A \sqsubseteq\left(P^{\prime} / P\right) A ; R
$$

where $\left(P^{\prime} / P\right) A$ stands for a substitution of each occurrence of $P$ by $P^{\prime}$ in the statements of $A$, and $R$ is an abstraction relation.

\section{Resolving Conflicts between Events and the Sys- tem State Space}

RULE 6: If $E$ is an input (output) event of working object $W O_{\text {whole }}$ then an extension of $E$ by some parameter of type $T$ implies an extension of the system state space by introduction of a property $T$.

$$
\begin{gathered}
E(. .) \sqsubseteq E(. ., T, . .) \Rightarrow \\
W O_{\text {whole }} \sqsubseteq+W O_{\text {whole }}^{\prime} \wedge \quad \Sigma_{w o^{\prime}}=\Sigma_{w o} \times \Sigma_{T}
\end{gathered}
$$

Note: If $\Sigma_{E^{\prime}} \subseteq \Sigma_{E}$ (a reduction) - there is no conflict with the system state space.

\section{Resolving Conflicts between Pre-, Post-conditions, and Updates}

A semantic refinement takes place when pre-, postcondition, or update statement of an action is changed. The equation (12) relates pre-, postcondition, and update. Precondition Pre can be calculated as a weakest precondition $w p(A, P o s t)$ that guarantees termination of any execution of $A(\langle U\rangle)$ in the final state that satisfies Post. Postcondition Post can be calculated as a strongest postcondition $s p(A, P r e)$ respectively. An update $U$ cannot be resolved by calculation and requires a modeler's decision.

Conflicts between pre-, post-conditions, and updates indicate that semantic refinement is incorrect. Correctness of semantic refinement is formulated in Definition 4.

\section{Resolving Conflicts between an Activity and its Component Actions}

Behavior distribution refinement stands for an activity definition (Figure 3). This requires a set of component actions and action ordering provided by modeler.

RULE 7: If an activity $A c$ is a behavioral distribution refinement of an action $A$, and $A_{1}, . . A_{t}$ - are component actions, see (13), then:

1. Events $E_{I n_{i}}$ and $E_{\mathrm{Out}_{i}}$ of component actions are defined based on the modeler's decision, providing (15) holds;

2. Preconditions $\mathrm{Pre}_{i}$ of component actions are defined either by propagation or based on modeler's decision, providing $\rho_{\text {pre }}$ for the given action ordering (14) is defined;

3. Updates $U_{i}$ and/or postconditions Post $t_{i}$ are defined based on modelers decision, providing $\rho_{u}, \rho_{\text {post }}$ for the given invocation order (14) are defined, and (12) holds for each component action;

Example8. Figure 3 illustrates the propagation of a behavior distribution refinement. Component actions GetFName, GetLName, and SaveData, their ordering, and set of input events are provided by a modeler. By refinement propagation, we define the preconditions for AddFName and AddLName component actions. Specification of the postcondition is the modeler's decision (here SaveData).

Without loss of generality, any action in SEAM specification can be seen as a component action of some abstract activity $A c_{\text {parent }}{ }^{3}$ Thus, the refinement of the behavior by an extension is considered as an introduction a new component to this abstract activity, whereas a reduction stands for elimination of some component from it. Rule 6, applied for the $A c_{\text {parent }}$, specifies the propagation of these refinements.

Both extension and reduction of the system's behavior have to preserve the semantics of the rest of the specification. Put it in other terms, modeler needs to guarantee that the system will work 'at least as well as before' in presence of new actions/activities or after removing any of them. This follows from the Definition 1 .

\footnotetext{
${ }^{3}$ System life cycle is the most abstract activity system performs from the moment of putting in operation (startup) till the end of functioning (shutdown). Any action or activity is a part of the life cycle.
} 


\section{Incorrectness}

RULE 8: Refinement propagation is impossible if the correctness of initial refinement is not provable.

\section{Summary}

In this section we provide definitions for the refinement correctness and formulate eight rules of refinement propagation. Refinement steps often represent combinations of refinements. For such a combination (7) and (8) hold.

We demonstrate that a significant part of specification elaboration can be done by calculation. We also specify the situations, when intervention of the modeler is necessary. Formal proof of soundness and completeness of the refinement propagation technique is an important issue. This makes a topic of our current research.

For the sake of brevity, some technical details have been omitted. For more explanations, please, contact the authors.

\section{RELATED WORK}

The foundations in mathematical logic are extensively used to formalize specifications and refinement techniques for program constructions. Woodcock and Davies (1996) present the method of software specification development called Z. Börger and Stärk (2003) introduce the Abstract State Machine method of abstract refinable system specifications. Refinement formalization for object-oriented programs using refinement calculus is presented by Mikhajlova and Sekerinski (1997), Back et al. (2000), and Michajlova (1998). Back(2005) proposes a method of incremental software construction using refinement diagrams. Here refinement calculus is used as a logic for reasoning about software systems and their evolution.

In the domain of visual languages, evolutionary specifications of ADORA are provided with refinement calculus semantic (Xia and Glinz, 2004). The transition between model views requires a representation consistency that is guaranteed by the application of refinement calculus. Muskens, in (Muskens et al., 2005), focuses on the problem of consistency checking between software views, expressed as UML diagrams. The approach in (Muskens et al., 2005) is based on verification of obligation and constraint rules using relation partition algebra. In contrast to these approaches, refinement propagation technique for SEAM black box specifications focuses on preservation of semantic correctness for visual specifications.

Baar and Marcović (2006) introduce a proof technique for the semantic preservation of refactoring rules for UML class diagrams and OCL constraints.
Refactoring can be considered as a specific form of refinement. We believe that the refinement propagation technique can be equally used to support automated refactoring of SEAM specifications. In our work, action constraints in SEAM (pre-, post- conditions, and updates) are specified using some meta-language. Alternatively, they can be expressed in OCL.

Pons (2006) presents the OCL-based technique and a tool support for UML and OCL model refinement. Object- $Z$ is an underlying theory for refinement verification. The authors discuss the refinement patterns and formulate the refinement conditions for these patterns in OCL language(OCL, 2003). Similarly, our technique considers several standard refinement types that can be identified with patterns. In addition, we define conflict situations, caused by these refinement types and explore the idea of refinement propagation.

\section{CONCLUSION}

In this work we formalize the notion of refinement and its correctness for visual specifications using refinement calculus (Back and von Wright, 1998). Based on this formalization, we define the refinement propagation technique for semiautomated specification construction. Initiated by modeler's design decision, correct refinement of the visual specification can be:

- automatically calculated, based on refinement propagation,

- calculated based on supplementary information from the modeler,

- recognized as impossible to calculate.

Eight rules of refinement propagation address possible conflicts between SEAM specification elements, caused by refinements. Figure 4 summaries the appli-

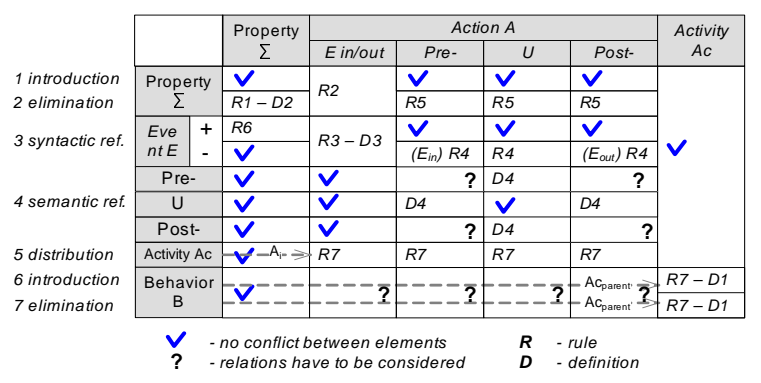

Figure 4: Refinement propagation rules. Summary.

cability of our technique. The leftmost column enumerates the refinements. Each row denotes conflicts between elements, caused by the respective refinement, and solutions for these conflicts. For example, 
an introduction of a property (row 1) causes no conflict with action preconditions (column 3), whereas property elimination (row 2) may cause such a conflict (column 3). This conflict can be resolved applying a propagation rule 5 .

Refinements may cause conflicts by breaking property-property and action-action relations in SEAM (Figure 1). Formalization, refinement, and refinement propagation for these relations is out of the scope of this paper. We put a question mark in the summary table to specify these cases.

We consider the refinement propagation technique as an efficient step towards computer-aided construction of visual specifications. Application of this technique in the form of a modeling tool is one of our on-going projects.

\section{REFERENCES}

Baar, T. and Marković, S. (2006). A graphical approach to prove the semantic preservation of UML/OCL refactoring rules. In Ershov Memorial Conference, Lecture Notes in Computer Science.

Back, R.-J. (1978). On the Correctness of Refinement Steps in Program Development. PhD thesis, bo Akademi, Department of Computer Science, Helsinki, Finland. Report A-1978-4.

Back, R.-J. (1989). Changing data representation in the refinement calculus. In 22nd Hawaii International Conference on System Sciences, pages 231-242. IEEE.

Back, R.-J. (2005). Incremental software construction with refinement diagrams. In Broy, Gunbauer, H. and Hoare, editors, Engineering Theories of Software Intensive Systems, NATO Science Series II: Mathematics, Physics and Chemistry, pages 3-46. Springer, Marktoberdorf, Germany.

Back, R.-J., Mikhajlova, A., and von Wright, J. (2000). Class refinement as semantics of correct object substitutability. Formal Aspects of Computing, 12(1):18-40.

Back, R.-J. and von Wright, J. (1998). Refinement Calculus: A Systematic Introduction. Springer-Verlag. Graduate Texts in Computer Science.

Börger, E. (2003). The asm refinement method. Formal aspects of computing.

Börger, E. and Stärk, R. (2003). Abstract State Machines: A Method for High-Level System Design and Analysis. Springer-Verlag.

Broy, M. (1993). Interaction refinement-the easy way. In Program Design Calculi: Proceedings of the 1992 Marktoberdorf International Summer School. Springer-Verlag.

Dijkstra, E. W. (1971). Notes on structured programming. In Structured Programming. Academic Press.

Mikhajlova, A. (1998). Consistent extension of components in presence of explicit invariants. In Workshop on Component-Oriented Programming (WCOP'98), ECOOP'98. TUCS General Publication Series.
Mikhajlova, A. and Sekerinski, E. (1997). Class refinement and interface refinement in object-oriented programs. In FME '97: Industrial Applications and Stengthened Foundations of Formal Metohds, volume 1313, pages 82-101. Springer.

Miller, J. (1995). Living Systems. University of Colorado Press.

Morgan, C. and Gardiner, P. H. B. (1990). Data refinement by calculation. Acta Informatica, 27(6):481-503.

Muskens, J., Bril, R. J., and Chaudron, M. R. V. (2005). Generalizing consistency checking between software views. In WICSA, pages 169-180.

OCL (2003). OCL 2.0 Final Adopted Specification. OMG.

Pons, C. (2006). Heuristics on the definition of UML refinement patterns. In SOFSEM, pages 461-470.

RM-ODP (1995). Reference model of open distributed processing part 1. Draft International Standard (DIS). Helsinki, Finland.

UML (2007). Unified Modeling Language (UML), version 2.1.1. OMG, www.omg.org.

Wegmann, A. (2003). On the systemic enterprise architecture methodology (seam). In International Conference on Enterprise Information Systems (ICEIS).

Wegmann, A. and Naumenko, A. (2001). Conceptual modeling of complex systems using an rm-odp based ontology. In EDOC, pages 200-211.

Weinberg, G. M. (1975). An Introduction to General Systems Thinking. New York: Wiley \& Sons.

Wirth, N. (1971). Program development by stepwise refinement. Communications of the ACM, 14:221-227.

Woodcock, J. and Davies, J. (1996). Using Z. Prentice Hall.

Xia, Y. and Glinz, M. (2004). Extending a graphic modeling language to support partial and evolutionary specification. apsec, 00:18-27. 